\title{
盗蜜行为在植物繁殖生态学中的意义
}

\author{
张彦文 ${ }^{1}$ 王 勇 $^{2}$ 郭友好 $^{1 *}$ \\ （1 武汉大学生命科学学院, 武汉 430072）（2 陕西理工学院生物系, 陕西汉中 723001)
}

\begin{abstract}
摘 要 在动植物的相互关系中, 盗蜜行为被认为是一种不同于普通传粉者的非正常访花行为。动物之所以要采 取这种特殊的受食策略, 有假说认为是由访花者的口器和植物的花部形态不匹配造成的, 也有认为是盗蜜行为提 高了受食效率从而使盗蜜者受益。在盗蜜现象中, 盗蜜者和宿主植物之间的关系是复杂的。盗蜜对宿主植物的影 响尤其是对其繁殖适合度的影响归纳起来有正面、负面以及中性 3 类。与此同时, 盗蜜者的种类, 性别及其掠食行 为差异不仅与生境因素密切相关, 而且会对宿主植物的繁殖成功产生直接或间接的影响。另外, 盗蜜者的存在无 疑对其它正常传粉者的访花行为也产生一定的影响，从而间接地影响宿主植物的繁殖成功，而植物在花部形态上 也出现了对盗蜜现象的适应性进化。作者认为, 盗蜜是短嘴蜂对长管型花最有效的一种掠食策略, 它不仅增加了 盗蜜者对资源的利用能力, 而且由于盗蜜对宿主植物繁殖成功的不同的影响使其具有调节盗蜜者和宿主之间种群 动态的作用，两者的彼此适应是一种协同进化的结果。
\end{abstract}

关键词 植物繁殖生态学 盗蜜现象 宿主植物 正常传粉者 繁殖适合度

\section{THE EFFECTS OF NECTAR ROBBING ON PLANT REPRODUCTION AND EVOLUTION}

\author{
ZHANG Yan-Wen ${ }^{1}$ WANG Yong ${ }^{2}$ and GUO You-Hao ${ }^{1 *}$ \\ (1 College of Life Sciences, Wuhan University, Wuhan 430072, China) \\ (2 Shaanxi University of Technology, Hanzhong, Shaanxi 723001 , China)
}

\begin{abstract}
The relationship between plant and pollinator is considered as a mutualism because plant benefits from the pollinator's transport of male gametes. Nectar robbers are frequently described as cheaters in the plant-pollinator mutualism , because it is assumed that they obtain a reward (nectar) without providing a service (pollination). Nectar robbers are birds, insects, or flower visitors that remove nectar from flowers through a hole pierced or bitten in the corolla. Nectar robbing represents a complex relationship between animals and plants. Whether plants benefit from the relationship is always the controversial part in earlier studies. This paper is to review the recent literatures on nectar robbing and attempt to acquire an expanded understanding of the ecological and evolutionary roles that robbers play. Understanding the effects of nectar robbers on the plants that they visited and other flower visitors is especially important when one considers the high rates of robbing that a plant population may experience and the high percentage of all flower visitors that nectar robbers make to some species.

There are two standpoints in explaining why animals forage on flowers and steal nectar in an illegitimate behavior. One is that animals can only get food in illegitimate's way because of the mismatch of the morphologies of animals' mouthparts and floral structure. The other point of view argues that nectar robbing is a relatively more efficient, thus more energy-saving way for animals to get nectar from flowers. This is probably associated with the difficulty of changing attitudes that have been held for a long time. In the case of positive effect , the bodies of nectar robbers frequently touch the sex organs of plants during their visiting to the flowers and causing pollination. The neutral effect, nectar robbers' behavior may destruct the corollas of flowers, but they neither touch the sex organs nor destroy the ovules. Their behavior don' $t$ affect the fruit sets or seed sets of the hosting plant. Beside the direct impacts on plants, nectar robbers may also have an indirect effect on the behavior of the legitimate pollinators. In some circumstances, the change in pollinator behavior could result in improved reproductive fitness of plants through increased pollen flow and out-crossing.
\end{abstract}

Key words Plant reproductive ecology, Nectar robbing, Host, Legitimate pollinator, Reproductive fitness 
以动物传粉的植物及它们之间的互利关系代表 了自然界一类极为普遍的种间相互作用, 但对于一 种非正常的访花行为一一盗蜜现象来说, 情况却十 分复杂。盗蜜 (Nectar robbing) (或译为劫蜜) 行为是 特指昆虫、鸟类或其它访花者不是从花冠开口处进 入, 而是通过在花冠上直接打洞并从中取食花蜜的 行为 (Inouye，1983；Maloof，2000)。盗蜜现象广泛 存在于自然界中, 几乎遍布除南极洲以外的全世界 各大洲( Irwin \& Maloof, 2002)。具有这种行为的昆 虫、鸟类等称为盗蜜者 (Nectar robber), 有时哺乳动 物如松鼠也可以成为盗蜜者 (Deng et al ，2003)。 盗蜜者可以分为初级盗蜜者 (Primary nectar robber) (在花冠上打洞取食花蜜) 及次级盗蜜者 (Secondary nectar robber) (不在花冠筒上打洞, 而是使用初级盗 蜜者已经打好的洞取食花蜜)。常见的盗蜜者是蜂 类, 尤以熊蜂 (Bombus) 和木蜂 (Xylocopa) 为多, 在美 洲蜂鸟也是常见的盗蜜者。有些甲虫在花上打洞， 但以取食花粉为主, 不食或极少取食花蜜, 所以不将 其作为盗蜜者看待 (Utelli \& Roy，2001)。被盗蜜植 物 (Host) (或译为宿主植物) 主要是一些具长花管或 花上有距 (Spur) 的产蜜植物。截止目前, 已经记载 有盗蜜现象存在的植物有 59 个科 214 种 (Irwin \& Maloof，2002）,而且我们推测，还有许多具有盗蜜现 象的植物尚未被研究发现, 因为大多数具有长管状 花部结构的含蜜植物中, 均可能有盗蜜现象发生。 在盗蜜现象中, 当事者动物一方的盗蜜行为对另一 方即宿主植物的影响，尤其是对其繁殖适合度 (Fitness) 的影响，一直是人们颇感兴趣的问题之一。达 尔文认为盗蜜会对植物的繁殖适合度造成负面影 响,他曾写道“: All plants must suffer in some degree when bees obtain their nectar in a felonious manner by biting holes through the corolla." (Darwin , 1872)。但长 期以来，特别是近 20 年来，随着人们对该问题不断 的深入研究, 发现盗蜜现象对宿主植物的影响并非 如此简单, 盗蜜者的盗蜜行为对植物繁殖适合度的 影响是多种多样的, 其原因也极为复杂, 主要因盗蜜 者的种类和盗蜜对象以及其它条件的不同而不同， 至今还没有总结出一般性的规律 (Newman \& Thomson , 2005)。

本文结合了前人对盗蜜行为研究的结果和作者 的研究观察, 初步探讨这一问题在生态和进化上的 意义。本文主要讨论了以下几个问题 : 1) 盗蜜者和 宿主植物之间的利益关系，2)盗蜜者的行为对其它 正常传粉者访花行为的影响 ; ) 盗蜜对花部进化的
影响 ; ) 盗蜜者及其掠食行为差异与宿主植物之间、 生境之间的相互影响。由于昆虫是最常见的盗蜜 者 本文主要讨论盗蜜昆虫和宿主植物之间的关系。

\section{1 盗蜜者和宿主植物之间的关系}

动物与植物在自然界中的相互作用是广泛存在 的，我们已有的概念和经验对这种作用的理解只限 于几种类型的拜访者, 主要是食草动物、传粉者、种 子掠食者和散布者(Maloof \& Inouye，2000)。植物与 传粉者之间被认为是一种互利关系, 对植物的益处 来自于传粉者传送了花粉，使之结出种子留下后代， 而传粉者得益于植物提供的花粉、花蜜等作为食物 报酬。盗蜜者多属于非正常的访花者，它们与被盗 蜜植物之间的关系不如正常访花者与被拜访植物之 间的关系那样明了, 有时是很复杂, 甚至是很不一致 的，所以有关盗蜜者和被盗蜜植物之间关系的问题 引起了研究者广泛的兴趣。已有的证据显示(Waser \& Real , 1979 ; Guitian et al . , 1994 ; Morris , 1996 ; Irwin \& Brody , 1999 ;Maloof , 2001 ; Richardson , 2004), 动植物在盗蜜过程中更多地表现为互利关系, 只是 这种互利关系有时明显 (主要表现在对雌性适合度 的影响), 有时较为隐蔽复杂 (主要表现在对雄性适 合度的影响), 可能只在少数负面影响的例子中, 盗 蜜的利益仅属于盗蜜者, 但此时的植物应当在长期 的进化过程中产生出适应结构或机制, 这一点将在 下文中予以讨论。

\section{1 盗蜜的原因}

为什么某些昆虫发展出不同于正常传粉者的访 花行为而成为盗蜜者? 长期以来就有两种观点, 一 种观点认为由于形态的不匹配, 盗蜜者只能通过这 种非正常途径获取花蜜，在这种情况下，盗蜜者一般 都是短喙的蜂类, 被盗蜜的植物种类都是具有狭长 花冠或距的花型，这种形态上的不匹配导致盗蜜行 为的发生是易于理解的。与此同时, 盗蜜者在形态 结构上也具有适应于盗蜜行为的某些特征，如一些 具盗蜜行为的熊蜂种类较之另一些不具盗蜜行为的 熊蜂种类在口器上有更多的齿 (Toothed)。然而并不 是所有具有短喙的蜂类都是盗蜜者, 只是其中一些 种类有盗蜜行为, 而另一些种类则没有, 这点是令人 难以理解的。另一种观点认为盗蜜者在花基部打洞 取蜜要比从花正面取蜜节约大量时间，比正常访花 更有效率，Inouye (1980)的实验结果也证明，短喙型 熊蜂通过盗蜜方式摄取花蜜要比长喙熊蜂以正常方 式取蜜更有效率, 它们拜访单花的平均时间分别为 
4.6 和 $6.6 \mathrm{~s}$, 次级盗蜜者访花的效率则更高一些。 为了提高取蜜效率而盗蜜, 会涉及到这样一个问题, 即盗蜜者的盗蜜行为是本能还是后天学习来的? 有 实验说明, 盗蜜者的打洞行为是天生的, 但选择花冠 上的打洞位置的行为却是后天学习而来的 (Olesen, 1996 ; Arizmendi , 2001)。

\section{2 盗蜜对宿主植物的影响}

由非正常传粉者 (Illegitimate pollinator)进行的盗 蜜行为及其对植物繁殖成功的影响非常普遍, 而我 们对这种现象的真实生态意义却了解不多。尽管近 年来人们对盗蜜现象进行了比以往更为深入的研 究, 但仍有人对盗蜜现象常常有一种习惯性的片面 认识, 即盗蜜者会给宿主植物带来负面影响, 因为它 们只盗食花蜜而不进行传粉。这种习惯性的认识产 生的原因无非是以往的固有观念一时转变困难造成 的。根据近 20 年来人们对盗蜜现象的研究, 盗蜜对 被盗蜜植物繁殖适合度的影响大致可以划分为负面 的 ( Negative) 影响、正面的 (Positive) 影响和中性的 (Neutral) 影响 3 类 (Maloof, 2000 ; Newman \& Thomson , 2005)。

\subsection{1 负面影响}

盗蜜对植物适合度产生负面影响很早就被注意 到。在盗蜜具负面影响的研究中, 人们发现盗蜜者 本身不是传粉者, 且在盗蜜过程中由于在一定程度 上破坏了花部结构, 减少了花对传粉者的吸引力并 缩短了单花期, 或者损坏了胚珠或蜜腺。也可能由 于盗蜜引起了花内蜜量减少或留有气味标记而改变 了正常传粉者 (或译为合法传粉者) (Legitimate pollinator)的访花行为, 从而降低了花粉的输出 (雄性适 合度降低)和柱头上花粉的落置 (雌性适合度降低)

(Traveset et al. , 1998 ; Irwin \& Brody , 1999 ; Irwin , 2003)。如在 Ipomopsis aggregata 中, 用类似花粉的颜 料作标记, 探讨了在盗蜜过程中花粉的输出和接受 量的变化, 结果表明, 盗蜜明显降低了植物的雌雄适 合度, 同时由于熊蜂的盗蜜影响了蜂鸟传粉者的掠 食 (Forage) 行为, 使其较少访问被盗蜜的花, 导致结 实率下降 (Irwin \& Brody, 1999)。严重的盗蜜(盗蜜 率 $80 \%$ 以上) 会明显降低宿主的结实率和种子的产 量, 并认为导致这种影响是由于传粉者回避被盗蜜 植物产生的 ( Irwin , 2003 ; Dedej \& Delaplane，2004)。 Traveset 等 (1998) 对倒挂金钟属 Fuchsia magellanica 的研究表明, 盗蜜引起植物结实率降低 $20 \%$ 的原因 是由于熊蜂在盗蜜时刺破花冠并损坏了部分胚珠。 在 Linaria vulgaris 上的研究表明, 盗蜜减少了其它正
常访花者的访花, 减少了花粉输出量, 降低了雄性适 合度, 但并未减少种子产量, 即并未影响其雌性适合 度(Newman \& Thomson, 2005)。上述结论说明, 某些 盗蜜行为的确会降低宿主植物的繁殖适合度。另外 盗蜜后植物往往要再次分泌蜜汁来补偿, 这种投资 的增加在一定程度上会影响种子的产量和质量是不 难理解的 (Irwin \& Brody , 1999 , 2000)。

由于盗蜜者有时获得报酬而不提供传粉，甚至 可能破坏花的结构, 所以经常被描写成骗子 (Darwin , 1872; Inouye , 1983 ; Maloof \& Inouye , 2000 ; Richardson，2004）这种负面影响对植物是不利的， 两者之间的关系也不能说是互利的，那么这种不对 称的利益关系为什么能长期存在呢? 一个可能的原 因是这种盗蜜行为表面上降低了宿主植物的结实 率, 但由于其改变了正常传粉者的访花行为, 可能提 高了宿主后代的异交率(Fenster，1991)，所以其后代 的生活力可能得到了加强, 在这一点上植物并非没 有得到任何利益。

\subsection{2 正面影响}

显示盗蜜对植物的雌雄适合度产生正面影响的 研究发现, 某些盗蜜者本身在盗蜜过程中身体的某 些部位会接触花的性器官一一花药和柱头, 起到一 定的传粉作用（Waser \& Real，1979; Guitian et al.， 1994 ; Navarro，2000），这时的盗蜜者就是传粉体系 的一部分,Higashi 等 (1988) 将其称为 Robber-like pollinator", 我们将其译为 似盗蜜传粉者”，以供商 椎。如 Navarro (2000) 在对 Anthyllis vulneraria subsp. vulgaris (豆科)的研究中发现, 被盗蜜频率很高的花 较未被盗蜜的花结实率更高, 认为盗蜜者对植物繁 殖成功的影响是与盗蜜者的行为及花和花序的结构 有关。Zimmerman 和 Cook (1985) 对凤仙花属 Impatiens capensis、Maloof (2001) 对紫堇属 Corydalis caseana 的研究发现, 盗蜜行为可使花粉被输送到更 远的距离, 这样可能促进异交, 对种群发展是有利 的。

在盗蜜对宿主植物的繁殖适合度具正面影响的 例子中, 盗蜜者在盗蜜的同时也完成了传粉, 甚至有 些植物花期的主要传粉者即为盗蜜者, 亦有较高的 结实率 (Scott , 1989) ，尽管许多研究表明这些盗蜜者 的传粉效率都要低于正常传粉者 (Kendall \& Smith, 1976），但这种有传粉作用的盗蜜者实际上就是一种 传粉者，它们与植物间的利益关系也是互利对称的。

\subsection{3 中性影响}

有些研究显示盗蜜对植物雌雄适合度没有明显 
影响 (Guitian et al., 1993 ; Morris , 1996 ; Maloof , 2001 ）其原因可能是由于盗蜜者在对宿主植物的盗 蜜过程中，本身不具传粉作用又没有对传粉者造成 明显影响。盗蜜者的掠食行为虽然可能在一定程度 上破坏了花冠或花距, 但多不损伤蜜腺和胚珠, 也不 影响单花期。此外,正常的传粉并受精后的胚珠, 种 子发育过程中的败育以及种子被昆虫啃食可能对其 繁殖成功影响更大(Stout et al . 2000)。Olesen 和 Knudsen(1994)对紫堇属植物的研究表明,由于熊蜂 盗蜜行为在时间上的特异性，决定了紫堇花在被盗 蜜时, 大部分花已经受精甚至种子已经开始发育, 这 些花仍然产生花蜜并不马上调谢, 其作用是能够增 强居群景观上的整体效应以吸引更多的昆虫给后开 的花传粉。

\section{3 盗蜜者及其掠食行为的差异对植物繁殖适合} 度的影响

盗蜜者种类及性别不同都会引起盗蜜行为差 异, 不同种甚至不同宗系 (Series)的熊蜂在掠食行为 上均有差异, 盗蜜的方式和时间也有不同( Ranta， 1983 ; Villalobos \& Shelly，1996)。在马先蒿属 ( Pedicularis) 某种熊蜂的蜂后是主要的盗蜜者, 而工 蜂则兼有掠食花粉及盗蜜行为, Waser 和 Real (1979) 在研究木蜂对 Fouquieria splendens 的盗蜜时也提到 其雌雄个体的取食行为不同, 这种性别引起的行为 差异, 可能是由昆虫的社会分工不同或生态位分化 造成的(Fussell，1992)。此外，有些盗蜜者在掠食过 程中其身份经常发生改变, 可能一段时间作为盗蜜 者,而另一段时间又作为合法传粉者, 这是因为被子 植物有着多样化的繁殖策略, 其花的形态和开花物 候都会影响盗蜜者的行为, 例如, 许多单花期较短的 花在其整个花期都会提供花粉和花蜜, 而一些单花 期较长的花只在第一天提供报酬, 其后就不再提供 了; 另一些花错开它们的报酬提供时间, 首先提供花 粉，再提供花蜜或者反过来(Ranta，1983 ;Rathcke， 1988)。由于不同时间段提供不同报酬，这样在发育 的不同阶段可能会吸引不同的访花者, 某些昆虫就 会在不同的时间和空间表现出不同的掠食行为，它 们会及时地在采集花蜜和花粉之间转换角色以适应 植物产生花蜜和花粉之间的时间和空间差, 这样植 物被访问后必然出现不同的效应。熊蜂就经常扮演 着双重掠食者的角色，它们在掠食花蜜时即常以盗 蜜者的身份出现，在掠食花粉时又是传粉者 (Macior , 1966 ; Morris , 1996 ; Olesen , 1996)。例如, 紫堇 属植物 C. ambigua 和 C. cava 在开花初期只产生
花粉，熊蜂蜂后也总是从其花的前部采食花粉，经过 一段时间大多数花开始产生较多花蜜后蜂后才直接 到距上盗蜜(Olesen，1996；Arizmendi，2001)，在蓝铃 花( Mertensia paniculata) 中也会看到熊蜂随着花期的 不同阶段而在盗蜜与采集花粉之间频繁地转换角色 的现象 (Morris, 1996)。

\section{2 盗蜜对正常传粉者访花行为的影响}

盗蜜者除了作为似盗蜜传粉者对植物产生明显 的正效应以外, 还可能通过改变正常传粉者的访花 行为, 从而间接地影响宿主植物的繁殖适合度, 这种 潜在的生态结果已被研究, 例如, 熊蜂在访问一朵低 蜜量的花后会飞更远的距离访问下一朵花,在访问 一朵蜜量丰富的花后飞行较短的距离访问下一朵花 (Miller \& Travis , 1996) ,Zimmerman 和 Cook（1985)及 Maloof (2000) 在不同的植物上也都有实验结果证 明, 盗蜜增加了正常传粉者在花间的飞行距离。当 花中含蜜量低于某一临界值时, 传粉者就很少访问 这些花或花序 (Pyke，1982；Hodges，1985) ,盗蜜者 可能扮演着调节花及花序中蜜量多少的角色。如果 盗蜜者的存在是导致正常传粉者飞行距离增加的原 因 那就会使花粉流传播距离增加, 进而通过提高异 交率来增加植物的雌性适合度 (Gliddon \& Saleem , 1984 ; Fenster，1991），许多实验结果表明异交导致增 加结实率或提高后代的存活率 (Charlesworth \& Charlesworth , 1987 ; Husband \& Schemske , 1996)。此 外，如果蜜酬由于盗蜜而减少，正常传粉者可能很快 就离开该花序, 于是减少了同株异花传粉, 并且增加 了花粉散布距离 (Klinkhamer \& de Jong，1993) ,这一 点对那些可以自交又有许多花在一个花序上的植物 尤其重要。盗蜜除了改变正常传粉者花间飞行距离 和访花数量外, 还可能改变正常传粉者花费在每朵 花上的时间, 被访花上含有大量的蜜会导致访花时 间加长, 进而使该花柱头落置大量花粉 (Thomson \& Plowright, 1980 ; Feisinger, 1983 ; Lanza et al. , 1995 ）而盗蜜者减少了蜜量水平，于是缩短了单次 访花时间 (Zimmerman \& Cook , 1985 ; Thomson , 1986 ; Maloof , 2000) , 其结果可能导致落置在柱头上的花 粉量减少 影响结实率, 这可能算做负效应, 不过, 传 粉者在每朵花上花费的时间缩短会使其在单位时间 内访问更多的花, 从这个角度看对植物总的繁殖成 功可能还是有益的(Maloof \& Inouye，2000)。

\section{3 盗蜜现象对花部形态进化的影响}

早期的研究一般多注意盗蜜对植物产生的负面 
影响 较少注意这种现象的积极一面, 随着对盗蜜现 象研究的不断深入, 人们已越来越多地注意到盗蜜 现象所带来的正面效应可能超过它的负面效应。如 上所述, 盗蜜者与盗蜜对象之间在大多数情况下是 一种互利关系, 只是这种非正常访花行为对植物的 繁殖适合度总是产生不同的影响, 至少表面上是这 样, 这一点常常令人疑惑。不过, 已有的研究和观察 表明, 产生这种现象的原因主要是盗蜜者本身行为 特点和掠食行为不同决定的 (Morris, 1996 ; Olesen, 1996 ; Arizmendi , 2001)。

人们一般接受这种观点, 传粉者可能通过选择 某种花(如形状、花色或其它特征)而影响花的进化 (Pyke，1982），如果盗蜜者的盗蜜行为对宿主植物的 适合度有影响, 那么盗蜜者就可能作为一种选择的 媒介对花部进化起作用, 但对这种作用的研究显然 被忽视了。一般说具长花管和距的花易被盗蜜, 而 长花管和距被选择是由于具长喙的传粉者受迫于这 种结构而使柱头落置更多的花粉 (Nilsson，1988)。 在花管长度达到某一值而影响正常传粉者传粉时, 盗蜜者的选择作用就会出现, 来自盗蜜者的选择作 用可能是直接的 (作为传粉者), 也可能是间接的 (影 响正常传粉者的访花行为), 例如, 如果本地区的正 常传粉者不能取食长花管或花距底部的蜜时，它们 可能会转到其它种类的花上掠食, 至少到蜜量积累 到一定水平后, 它们才能取到花蜜, 但此时如果有盗 蜜者, 它们能取食这些底部的蜜, 那么底部的花蜜总 达不到使正常传粉者能够获取的水平。现在假设这 些盗蜜者还收集花粉，在这个过程中起到传粉作用， 在这种情况下, 盗蜜者很可能被作为选择的媒介, 起 到正效应。对于具长花管的花来说, 由于长花管不 仅不阻碍盗蜜者取蜜或传粉, 而且盗蜜者在选择盗 蜜对象时更可能或喜欢访问长花管的花, 因为这种 花中的花蜜最有可能未被正常传粉者取走 (Maloof \& Inouye ,2000)。Roubik 等 (1985) 发现 Quassia amara 的花管长度在被高频率盗蜜的居群比隔离未被盗蜜 的居群更长一些。Fouquiera splendens 的花象其它花 一样在形态上有许多变异, Waser 和 Real (1979)相信 这些变异可能是适应于木蜂那样的盗蜜者传粉的。 还有人注意到, 如果植物产生的蜜量除了满足盗蜜 者外, 还余有部分给其它传粉者, 这样的植物可能留 下最多的后代(Barrows，1980；Pyke，1982) ,在 Quassia amara 中,被盗蜜严重的居群可能产生平均 45.3 $\mu \mathrm{l}$ 蜜, 而在末被盗蜜居群是 $30.8 \mu \mathrm{l}$,Morris (1996) 认 为这种高盗蜜率对应于高产蜜量是一种协同进化
(Co-evolution)。盗蜜如果对植物有持续的负面影 响, 植物应该会演化出防御结构和机制, 如有些植物 演化出花外蜜腺，吸引蚂蚁等来抵御盗蜜者的侵袭 (Elias \& Gelband，1975)。结构适应或许是一种低成 本的防止盗蜜的方式，如 Thunbergia grandiflora 的花 组织加厚就是一种使盗蜜者打消主意的方式，增强 增大萼片或苞片是另一种可供选择的方式,如在 Lonicera involucrata 中 (Proctor et al ，1996)。另外， 产生密集的花序代替单花也是避免盗蜜的方式, 至 于产生特殊的化学抑制物来防止盗蜜也是可能的， 但成本可能较高 (Inouye，1983)。对多数植物来说， 盗蜜并未对其产生足够的负面影响，因此也就不必 演化出防御结构和机制。相反，如果盗蜜对其有正 面影响, 那么植物尤其是花部特征一定会向着适应 方向演化。

\section{4 植物及生境对盗蜜行为的影响}

发生盗蜜行为最频繁的植物一般为那些具有形 态上的限制、使得盗蜜者口器无法达到花冠或距的 基部吸取花蜜的植物。不同的植物具有不同的花部 构成 (Floral design) 和开放形态, 这种多态性 (Polymorphism) 影响盗蜜行为, 盗蜜者对其有选择性 (Navarro，2001；Olesen \& Knudsen，1994），大花序可 以吸引更多的访花者包括盗蜜者 (Ohara \& Higashi， 1994）花色较淡或蜜中糖浓度较低的个体不利于吸 引访花者, 植物个体间的花色和气味差异以及花蜜 中糖的浓度都对盗蜜者行为产生一定影响 (Goulson et al . , 1998) 花距的特殊形态可决定盗蜜熊蜂对蜜 的接受性(Kato et al. , 1991)。此外, 盗蜜者的盗蜜 行为还与被盗蜜对象的生境有关, 对生活在不同海 拔高度的乌头属植物 Aconitum napellus 的研究表明， 高海拔 $(2000 \mathrm{~m}$ ) 地区生长的乌头花由短喙 (Shorttongued) 熊蜂盗蜜造成的损坏较严重, 而低海拔 (500 $\mathrm{m})$ 地区生长的乌头则较少受到熊蜂盗蜜的影响 (Utelli \& Roy, 2001)。另外, 喜欢盗蜜的短喙熊蜂多 在开阔地带或大的斑块上活动, 而中长喙 (Middleand-long-tongued) 的熊蜂则被迫到小斑块上活动, 这 种现象在 Symphytum officinalis 和 Aconitum napellus 中 极为明显 (Sowig, 1989)。盗蜜者的行为受植物和环 境影响, 这种影响反过来又可能导致不同生境或斑 块中植物适合度的改变, 进而影响该植物的种群动 态。

\section{5 展 望}

动植物在生态系统中有着千丝万缕的联系, 尤 
其是昆虫与植物间的关系更是千变万化, 昆虫与植 物间的协同进化不能不说是大自然的杰作 (钦俊德, 1987)。盗蜜者的盗蜜行为究竟对植物的适合度或 繁殖成功有什么样的生态和进化意义, 盗蜜者、正常 访花者与植物间在微观进化 (Microevolution)中的某 些微妙关系人们还在推测和不断探索中, 这方面的 研究还很少, 可能会成为该方向下一步研究的重点, 但经过多年来的研究，作者认为至少可以从两方面 得出以下认识。

对盗蜜者来说, 这种行为可以更有效地掠食。 熊蜂能从花管或距中花蜜的紫外反射光谱判断出花 中蜜的体积和糖含量高低 (Throp et al . , 1975) , 直接 盗蜜要比从花前部正常取蜜便利得多, 这种节省能 耗的取食行为对其自身有明显的益处。其次，不同 喙长的熊蜂选择不同的取食方式，短喙的种类适合 于盗蜜, 而长喙的种类更适合于正常采蜜, 蜂鸟也是 这样( Irwin \& Brody，2000)。另外，两者在选择拜访 对象的居群斑块和空间上也有所不同, 这明显表现 出不同生物以及不同种类在生态系统中占据不同的 生态位, 有利于生物对资源的合理分配和充分利用。

对被盗蜜植物来说, 事实上多数情况下并未产 生负面影响, 盗蜜在某种程度上有一定的传粉作用, 甚至在紫堇属 C. a a bigua 中有近 60\%的花是通过 盗蜜者访问结实的 (Olesen, 1996)。多项实验也显 示, 盗蜜者行为及对其它访花者行为的影响能使花 粉流的传播距离更远, 减少了同株异花传粉的几率, 增加异交和变异以适应环境 (Irwin, 2003)。盗蜜行 为的负面影响在某些植物中也是显而易见的, 严重 的盗蜜会减少正常传粉者的访问，从而降低了植物 的雌雄适合度。应该指出的是，不同的作者在不同 的植物上或在同一植物不同环境下得出不同甚至相 反的结论也不足为奇, 因为昆虫与植物间的关系本 身就是复杂多变的。

值得注意的是, 长期以来我国学者对这一研究 方向重视不够, 缺乏必要的、深入系统的研究, 许多 名词只是零散地出现在各类文章中或者没有很好的 中文释义。可喜的是近两年来邓晓保等 (Deng et $a l$. , 2003; 邓晓保等, 2005) 在国家自然基金的资助 下已经对此展开了探索并取得进展, 预计今后会有 更多的人对此投入兴趣并展开研究。

总之, 盗蜜行为作为一种复杂的生态现象, 可能 对植物与访花者之间的关系产生深刻影响。而对诸 如盗蜜者不同物种之间及其与传粉者之间的竞争关 系 花的形态及植物与访花者之间的协同进化, 以及
其中所包含的进化生态学意义, 人们还在探索中。 但可以肯定的是, 盗蜜者、传粉者以及植物之间必定 有着某种微妙的关系，它们都是动植物互利关系整 体的一部分, 只是互利双方在利弊的天平上对某些 物种来说可能是不平衡的。如果我们仅简单地认为 利益倾向于盗蜜者的话, 可能也不尽然,且不说盗蜜 对一些植物有一定正效应。但就生态平衡来说，如 果盗蜜对植物的适合度破坏达到一定程度，必然要 使该植物的种群数量下降, 这样环境中能提供给盗 蜜者的能量资源就会减少和重新分配;反之则必然 影响盗蜜者本身的种群动态。对于这种复杂的生态 关系, 目前我们的认识程度还很有限。

\section{参 考 文 献}

Arizmendi MC (2001). Multiple ecological interactions: nectar robbers and hummingbirds in a highland forest in Mexico. Canadian Journal of Zoology, 79, 997 - 1006.

Barrows EM (1980). Robbing of exotic plants by introduced carpenters and honeybees in Hawaii, with comparative notes. Biotropica, 12, 23-29.

Charlesworth D, Charlesworth B (1987). Inbreeding depression and its evolutionary consequences. Annual Review of Ecology and Systematics, 18, $237-268$.

Darwin C (1872). The Effects of Cross and Self-Fertilization in the Vegetable Kingdom. Murray, London, UK.

Dedej S, Delaplane KS (2004). Nectar-robbing carpenter bees reduce seed-setting capability of honey bees (Hymenoptera: Apidae) in rabbiteye blueberry, Vaccinium ashei, 'Climax' . Environmental Entomology, 33, 100 - 106.

Deng XB (邓晓保), Ren PY (任盘宇), Li QJ (李庆军) (2005). Nectar secretion patterns, floral visitor behavior and their impacts on fruit and seed sire of Alpina blepharocalyx. Acta Phytoecologica Sinica (植物生态学报), 29, 274-280.（in Chinese with English abstract)

Deng XB, Ren PY, Gao JY, Li QJ (2003). The striped squirrel ( Tamiops swinhoei hainanus) as a nectar robber of ginger (Alpinia kwangsiensis ). Biotropica, 36, 633-636.

Elias TS, Gelband H (1975). Nectar: its production and functions in trumpet creeper. Science, 189, $289-291$.

Feisinger P (1983) . Coevolution and pollination. In: Futuyma DJ, Slattkin M eds. Coevolution. Sinauer Associates, Sunderland, Masschusetts, $285-319$.

Fenster CB (1991). Gene flow in Chamaecrista fasciculata (Leguminosae). Evolution, 45, 398-422.

Fussell M (1992). Diurnal patterns of bee activity, flowering, and nectar reward per flower in tetraploid red clover. Newzeland Journal of Agricultural Research, 35, $151-156$.

Gliddon C, Saleem M (1984). Gene-flow in Trifolium repens-an expanding genetic neighborhood. In: Jacquard P, Heim G, 
Antonovics J eds. Genetic Differentiation and Dispersal in Plants. Springer-Verlag, New York, USA, 293 - 309.

Goulson D, Hawson SA, Stout JC (1998). Foraging bumblebees avoid flowers already visited by conspecifics or by other bumblebee species. Animal Behaviour, 55, 199 - 206.

Guitian J, Sanchez JM, Guitian P (1994). Pollination ecology of Petrocoptis grandiflora Rothm. (Caryophyllaceae) : a species endemic to the north-west part of the Iberian Peninsula. Botanical Journal of the Linnean Society, 115, $19-27$.

Guitian P, Guitian J, Navarro L (1993). Pollen transfer and diurnal versus nocturnal pollination in Lonicera etrusca. Acta Oecologica, 14, 219-227.

Higashi S, Ohara M, Arai H, Matsuo K (1988). Robber-like pollinators: overwintered queen bumblebees foraging on Corydalis ambigua. Ecological Entomology, 13, 411 - 418 .

Hodges CM (1985) . Bumble bee foraging: the threshold departure rule. Ecology, 66, $179-187$.

Husband BC, Schemske DW (1996). Evolution of the magnitude and timing of inbreeding depression in plants. Evolution, 50, 54 -70 .

Inouye DW (1980). The terminology of floral larceny. Ecology, $61,1251-1252$.

Inouye DW (1983). The ecology of nectar robbing. In: Bentley B, Elias T eds. The Biology of Nectaries. Columbia University Press, New York, USA, 153 - 173.

Irwin RE (2003) . Impact of nectar robbing on estimates of pollen flow: conceptual predictions and empirical outcomes. Ecology, $84,485-495$.

Irwin RE, Brody AK (1999). Nectar-robbing bumble bees reduce the fitness of Ipomopsis aggregata (Polemoniaceae). Ecology, $80,1703-1712$.

Irwin RE, Brody AK (2000). Consequences of nectar robbing for realized male function in a hummingbird-pollinated plant. Ecology, 9, $2637-2643$.

Irwin RE, Maloof JE (2002). Variation in nectar robbing over time, space, and species. Oecologia, 133, 525-533.

Kato M, Itino T, Hotta M, Inoue T (1991). Pollination of four Sumatran Impatiens species by hawkmoths and bees. Tropics, 1, $59-73$.

Kendall DA, Smith BD (1976). The pollinating efficiency of honeybee and bumblebee visits to flowers of the runner bean Phaseolus coccineus L. Journal of Applied Ecology, 13, 749 - 752.

Klinkhamer PGL, de Jong TJ (1993) . Attractiveness to pollinators: a plant's dilemma. Oikos, 66, 180-184.

Lanza J, Smith GC, Sack S, Cash A (1995). Variation in nectar volume and composition of Impatiens capensis at the individual, plant, and population levels. Oecologia, 102, 113 - 119 .

Macior LW (1966). Foraging behaviour of Bombus (Hymenoptera: Apidae) in relation to Aquilegia pollination. American Journal of Botany, 53, $302-309$.

Maloof JE (2000). Reproductive biology of a North American sub- alpine plant: Corydalis caseana A. Gray ssp. brandegei ( $\mathrm{S}$. Watson) G. B. Ownbey. Plant Species Biology, 15, 281 - 288.

Maloof JE (2001). The effects of a bumble bee nectar robber on plant reproductive success and pollinator behavior. American Journal of Botany, 88, 1960 - 1965 .

Maloof JE, Inouye DW (2000) . Are nectar robber cheaters or mutualists? Ecology, 81, 2651-2661.

Miller TE, Travis J (1996). The evolutionary role of indirect effects in communities. Ecology, 77, 1329-1335.

Morris WF (1996) . Mutualism denied? Nectar-robbing bumble bees do not reduce female or male success of bluebells. Ecology, 77, $1451-1462$.

Navarro L (2000) . Pollination ecology of Anthyllis vulneraria subsp. vulgaris (Fabaceae): nectar robbers as pollinators. American Journal of Botany, 87, 980-985.

Navarro L (2001) . Reproductive biology and effect of nectar robbing on fruit production in Macleania bullata (Ericaceae). Plant Ecology, 152, 59 - 65 .

Newman DA, Thomson JD (2005) . Effects nectar robbing on nectar dynamics and bumblebee foraging strategies in Linaria vulgaris (Scrophulariaceae). Oikos, 110, 309-320.

Nilsson LA (1988). The evolution of flowers with deep corolla tubes. Nature, 334, $147-149$.

Ohara M, Higashi S (1994). Effects of inflorescence size on visits from pollinators and seed set of Corydalis ambigua (Papaveraceae). Oecologia, 98, 25-30.

Olesen JM (1996). From naivete to experience: bumblebee queens (Bombus terrestris) foraging on Corydalis cava (Fumariaceae). Journal of Kansas Entomology Society, 69, $274-286$.

Olesen JM, Knudsen JT (1994). Scent profiles of flower colour morphs of Corydalis cava (Fumariaceae) in relation to foraging behaviour of bumblebee queens (Bombus terrestris). Biochemical Systematics and Ecology, 22, $231-237$.

Proctor M, Yeo P, Lack A (1996). The Natural History of Pollination. Timber Press, Oregon.

Pyke GH (1982) . Foraging in bumblebees: rule of departure from an inflorescence. Canadian Journal of Zoology, 60, 417 - 428 . Qin JD (钦俊德) (1987). The Relationships Between Insects and Plants (昆虫与植物的关系). Science Press, Beijing, 192 201. (in Chinese)

Ranta E (1983) . Foraging differences in bumblebees. Annual Review of Entomology, 49, 17 - 22 .

Rathcke B (1988). Flowering phonologies in a shrub community: competition and constraints. Ecology, 76, 975-994.

Richardson SC (2004). Are nectar-robbers mutualists or antagonists? Oecologia, 139, $246-254$.

Roubik DW, Holbrook NM, Parra VG (1985). Roles of nectar robbers in reproduction of the tropical treelet Quassia amara (Simaroubaceae). Oecologia, 66, $161-167$.

Scott PE (1989). Ecological Consequences of Varieties Pollinator Availability: Ocotillo, Carpenter Bees, and Hnmingbirds in Two 
Deserts. PhD Dissertation, Louisiana State University, Baton Rouge, Louisian, USA.

Sowig P (1989). Effects of flowering plant's patch size on species composition of pollinator communities, foraging strategies, and resource partitioning in bumblebees (Hymenoptera: Apidae). Oecologia, 78, $550-558$.

Stout JC, Allen JA, Goulson D (2000) . Nectar robbing, forager efficiency and seed set: bumblebees foraging on the self incompatible plant Linaria vulgaris (Scrophulariaceae). Acta Oecologica, $21,277-283$.

Thomson JD ( 1986). Pollen transport and deposition by bumble bees in Erythronium: influence of floral nectar and bee grooming. Journal of Ecology, 74, 329 - 342.

Thomson JD, Plowright RC (1980) . Pollen carryover, nectar rewards, and pollinator behavior with special reference to Diervilla lonicera. Oecologia, 46, 68-74.
Throp RW, Briggs DL, Estes JR (1975). Nectar fluorescence under ultraviolet irradiation. Science, 189, 476-478.

Traveset A, Willson MF, Sabag C (1998). Effect of nectar-robbing birds on fruit set of Fuchsia magellanica in Tierra Del Fuego: a disrupted mutualism. Functional Ecology, 12, 459 - 464 .

Utelli AB, Roy BA (2001). Causes and consequences of floral damage in Aconitum lycoctonum at high and low elevations in Switzerland. Oecologia, 127, 266-273.

Villalobos EM, Shelly TE (1996). Temporal and spatial variation in the foraging behavior of honey bees (Hymenoptera: Apidae) at Chinese violets. Florida Entomologist, 79, 398 - 407.

Waser NM, Real LA (1979). Effective mutualism between sequentially flowering plant species. Nature, 281, 670-672.

Zimmerman M, Cook S (1985). Pollinator foraging, experimental nectar-robbing and plant fitness in Impatiens capensis. American Midland Naturalist, 113, $84-91$.

责任编委 : 张大勇 责任编辑 :刘丽娟 